\title{
IMPLICACIONES DE LA «GIG-ECONOMY» EN LAS RELACIONES LABORALES: EL CASO DE LA PLATAFORMA UBER*
}

\section{Implications of the "gig-economy» in labor relations: The Case of the Uber Platform}

\author{
Maria Saenz de Buruaga Azcargorta \\ Universidad de Deusto \\ maria.saenzdeburuaga@gmail.com \\ http://dx.doi.org/10.18543/ed-67(1)-2019pp385-414
}

Recibido: 15.02 .2019

Aceptado: 21.06 .2019

\section{Resumen}

En un entorno laboral cada vez más digitalizado, proliferan en España nuevas formas de trabajo al margen del actual cuadro normativo. Así, plataformas de la «gigeconomy» como Uber, Deliveroo o Glovo cuentan cada día con más «microtrabajadores» dispuestos a prestar servicios y realizar pequeños encargos. Este artículo trata de analizar, partiendo del caso de la plataforma de transporte urbano Uber, la relación que une a las plataformas digitales y a aquellos que ofrecen sus servicios en éstas. En este sentido, se analiza a partir de un análisis pormenorizado de los rasgos definitorios de estas nuevas formas contractuales, la existencia de laboralidad a la luz de los últimos pronunciamientos administrativos, jurisprudenciales y doctrinales habidos hasta la fecha. Por último, se presentan soluciones regulatorias ad hoc para tratar de dar respuesta a los distintos intereses que se hallan en conflicto: por un lado, la

* Cómo citar / Citation 'Chicago-Deusto' (Autor-fecha / Author-date / Lista de referencias / Reference list entries): Saenz de Buruaga Azcargorta, Maria. 2019. «Implicaciones de la «gig-economy» en las relaciones laborales: el caso de la plataforma Uber». Estudios de Deusto 67, n. ${ }^{\circ}$ 1: 385-414. http://dx.doi.org/10.18543/ed-67(1)-2019pp385-414. 
necesaria protección de los prestadores de servicios y, por otro, su creciente popularidad y atractivo entre proveedores y usuarios.

\title{
Palabras clave
}

Economía colaborativa; plataformas digitales; precarización; Uber; falsos autónomos.

\begin{abstract}
The rise of the gig-economy is having a significant impact on job creation all across Europe. More and more young workers are being employed in platforms such as Uber, Deliveroo or Glovo, supplementing their part-time jobs to earn additional income. This paper aims at analizing, taking the taxi-hailing company Uber as a case study, the employment status of those who offer their services through these platforms. In this respect, the article carries out a detailed assessment of the defining features of these new emerging contracts, with a focus on determining whether an employment or independent contractor relationship exists on the basis of recent judgements and administrative doctrine. The last section of the paper presents some ad hoc regulatory proposals that could weigh up the conflicting interests involved: on one hand, the need of ensuring basic social protection for platform workers and, on the other hand, the rising popularity of the gig-economy from both offer and supply approaches.
\end{abstract}

\section{Keywords}

Sharing Economy; Platform workers; Uber; Precarity; false self-employee. 
Sumario: I. INTRODUCCIÓN. II. NuEVAS FORMAS DE PRESTACIÓN DE SERVICIOS EN LA ERA DIGITAL: LA «GIG-ECONOMY». 1. Concepto de "gig-economy». 2. "Microtrabajadores» de la "gig economy» y precariedad laboral. 3. Relación entre la "gig economy» y las E.T.T's. 4. Uber como manifestación de la "gig-economy» en España. III. SoBRE LA LABORALIDAD EN LAS RELACIONES ENTRE UBER Y SUS CONDUCTORES. 1. Concepto tradicional del trabajador por cuenta ajena. 1.1. Elementos definitorios de la relación laboral y su distinción frente al trabajo autónomo. 1.2. Flexibilización de la nota de subordinación y dependencia funcional. 2. Aplicación del test de laboralidad a los trabajadores de UBER. 2.1. Voluntariedad. 2.2. Retribución. 2.3. Subordinación o dependencia funcional. a) Establecimiento de concretas instrucciones e indicaciones sobre la forma de prestación de servicios. b) Toma de decisiones estratégicas y comerciales por parte de la empresa. c) Los conductores se hallan sujetos a un permanente sistema de control. 2.4. Ajenidad. a) Ajenidad en los frutos. b) Ajenidad en los riesgos. c) Ajenidad en el mercado. d) Conclusión. 3. Pronunciamientos administrativos, jurisprudenciales y doctrinales. 3.1. Administrativos: Informe de la Inspección de Trabajo y Seguridad Social Catalana. 3.2. Jurisprudenciales: Sentencia del Tribunal Central de Trabajo de Londres y dictamen de la Comisión de Trabajo de California. a) Sentencia del Tribunal Central de Trabajo de Londres. b) Dictamen del Comisión de Trabajo de California. 3.3. Doctrinales. 4. Problemas de encaje del nuevo tipo de trabajadores en el concepto tradicional de contrato de trabajo. IV. SOLUCIONES PARA DAR RESPUESTA A LOS NUEVOS RETOS LABORALES PLANTEADOS POR LA «GIG-ECONOMY». 1. La autorregulación. 2. Relación laboral especial. V. CONCLUSIONES. VI. BIBLIOGRAFIA.

\section{INTRODUCCIÓN}

La aplicación de las nuevas tecnologías de la información y la comunicación está conduciendo a la emergencia de nuevas formas de relaciones laborales. Así, a través de las plataformas virtuales, proliferan en España las contrataciones puntuales de personas dadas de alta en dichas plataformas para la realización de trabajos esporádicos. De hecho, según EUCoLab ${ }^{1}$, el $6 \%$ de la población española ya ofrece sus productos o servicios a través de estas redes. Ante esta mutación de los modelos productivos, se plantean nuevos retos laborales con los que, como en otras ocasiones ya ha hecho -por ejemplo, regulando el teletrabajo-, el Derecho del Trabajo debe lidiar.

${ }^{1}$ EUCOLAB. Cuestiones clave a las que se enfrenta la economía colaborativa en Europa. Bruselas: The European Collaborative Economy Forum, 2016. 
El objeto del presente trabajo es analizar, partiendo del caso de la plataforma de transporte urbano de pasajeros Uber, la relación que une a las plataformas digitales y a aquellos que ofrecen sus servicios en éstas. Para ello, tras una primera aproximación general al concepto de la «gig-economy» y a los trabajadores que en ella se emplean, se valorará la existencia de laboralidad entre la plataforma y sus conductores, estudiándose los pronunciamientos administrativos, jurisprudenciales y doctrinales habidos hasta la fecha. Por último, tras valorarse las posibles dificultades de encaje de este tipo de trabajadores dentro del concepto tradicional del contrato de trabajo, se destacarán algunas de las posibles soluciones que plantea la doctrina.

El principal motivo que me ha empujado a escoger esta temática ha sido el fuerte crecimiento que están experimentando las plataformas de la «gigeconomy». Así, no cabe duda de que estos modelos de negocio están repercutiendo en la economía de forma positiva. Sin embargo, conviene interesarse por los trabajadores que allí se ofrecen con el fin de fomentar un entorno reglamentario que, sin impedir el desarrollo de estas plataformas, proteja los intereses de sus usuarios.

En relación con la metodología empleada, cabe destacar que, dado que en España nos hallamos en la fase prerregulatoria de este fenómeno, el análisis sobre esta cuestión se ha concentrado mayoritariamente en el estudio de artículos científicos recientemente publicados por la doctrina española. Además, al tratarse de una cuestión que también ha sido tratada en el ámbito internacional -concretamente en California y Reino Unido-, también se ha hecho uso de los argumentos contenidos en estas resoluciones.

\section{NUEVAS FORMAS DE PRESTACIÓN DE SERVICIOS EN LA ERA DIGITAL: LA «GIG-ECONOMY»}

\section{Concepto de "gig-economy»"}

Una de las ventajas que ha traído consigo la emergencia de las nuevas tecnologías de la información y de la comunicación (NTIC) se refiere a la rebaja de los costes de transacción. Así, las empresas, a la hora de externalizar parte de sus actividades productivas, soportan muchos menores costes que antaño. Este cambio ha provocado que muchas organizaciones empresariales descentralicen todas aquellas actividades que no constituyen su core business, favoreciendo un entorno empresarial más competitivo en el que las estructuras

${ }^{2}$ HILL, Steven. «The Future of Work in the Uber Economy. Creating a Safety Net in a Multi-Employer World», Boston Review: Political and Literary Forum, http://bostonreview.net/us/steven-hill-uber-economy-individual-security-accounts (Última consulta 3 de mayo de 2017). 
organizativas son más reducidas y flexibles. Evidentemente, esta evolución ha supuesto un cambio que no solo ha afectado al tamaño de las organizaciones empresariales, sino también a las propias relaciones laborales que allí se desarrollan. En este sentido, cada vez resultan más numerosas las nuevas formas de trabajo existentes, nuevas formas, que llegan a modificar -incluso sustancialmente- las características propias del puesto de trabajo, las condiciones laborales de los trabajadores o las experiencias vitales en el trabajo.

En el ámbito anglosajón, la «gig-economy»-originariamente, «economía del pequeño encargo»- hace referencia al modelo de externalización productiva del nuevo entorno digital. Así, este término engloba a todas aquellas plataformas virtuales que, a través de la externalización de su actividad económica mediante trabajadores autónomos, están creando nuevas formas de prestación de servicios. Este modelo productivo presenta, según GINES I FABRELLAS ${ }^{3}$, las siguientes características.

En primer lugar, las plataformas virtuales dividen la prestación de servicios en tareas individuales o «microtasks». Esto es, en vez de ofrecer un servicio de forma integral, estas empresas de base tecnológica dividen el trabajo en tareas de corta duración, individuales e independientes. A modo de ejemplo, en el caso de Uber, en vez de ofrecer el servicio de transporte urbano de forma conjunta, ofrece trayectos individuales.

En segundo lugar, cada una de estas «microtasks» es externalizada a múltiples trabajadores autónomos. Así, más allá de externalizar con una única empresa o trabajador por cuenta propia el servicio de una forma más o menos estable, las nuevas tecnologías permiten la íntegra externalización de la actividad económica a un número elevado de trabajadores autónomos mediante un llamamiento o convocatoria abierta. El objetivo de estas plataformas es gozar de un número suficientemente elevado de prestadores de servicio para garantizar poder atender, en todo momento, a la demanda existente. Esto se denomina crowdsourcing.

En tercer lugar, las nuevas tecnologías permiten la contratación de trabajadores autónomos on-demand. Esto es, los trabajadores autónomos son contratados para la prestación del servicio en el momento preciso en que se produce la demanda o solicitud del servicio por parte del cliente y únicamente por la duración de ese servicio en concreto. Por consiguiente, estas plataformas virtuales, más allá de contratar a trabajadores autónomos para la prestación de servicios, se limitan a conectar en el tiempo y momento exacto de la realización del servicio con los proveedores inscritos en la plataforma,

${ }^{3}$ GINES I FABRELLAS, Anna. «Crowdsourcing: nueva modalidad de externalización productiva en el entorno digital. Reflexiones sobre su viabilidad jurídica.» Iuslabor, Asociación Catalana de Iuslaboralistas. Recuperado http://www.iuslabor.org/jornades-iseminaris/comunicacions/any-2017/ (Última consulta el 21 de abril de 2017). 
reduciéndose así drásticamente los costes empresariales asociados a períodos de inactividad o improductivos.

En este sentido, tal y como precisa RODRIGUEZ-PIÑERO ROYO ${ }^{4}$, la «gig-economy» no se refiere únicamente a las plataformas de economía colaborativa, en las que debe regir un verdadero espíritu de colaboración en el intercambio, sino que también abarca todas aquellas plataformas cuya razón de ser sólo implica la conexión directa entre prestador y usuario, evitando la intermediación de empresas o mercados. Paralelamente, el Comité de Regiones de la Unión Europea, en su informe de $2015^{5}$ sobre «El consumo colaborativo o participativo», calificó la «gig-economy» como aquel modelo en que se desarrollan «iniciativas basadas en trabajos esporádicos cuya transacción se hace a través del mercado digital».

Con todo, enfocando estas definiciones hacia la perspectiva del Derecho del Trabajo, la «gig-economy» reenviaría a todas aquellas situaciones laborales en las que se contrata a una persona a través de plataformas digitales para ejecutar trabajos esporádicos, en los que el trabajador aporta todo lo necesario para el desarrollo de la actividad.

\section{2. «Microtrabajadores» de la "gig economy» y precariedad laboral}

Tal y como reconoce la Organización Internacional del Trabajo (en adelante, O.I.T.) en su recomendación número $204^{6}$, existe un importante porcentaje de la fuerza de trabajo mundial que se gana la vida en la «economía informal». Precisamente, dentro de ese concepto, se encuentran aquellas plataformas que comprenden «actividades económicas desarrollados por trabajadores y unidades económicas que, en la legislación o en la práctica, están insuficientemente cubiertas por sistemas formales o no lo están en absoluto». Así, la «gig-economy» integraría el concepto de economía informal y, por ello, el Comité de Regiones de la Unión Europea urge la necesidad de regular un sector que ha reportado, sólo en 2015, más de 28 millones de euros a la economía europea. Entre los aspectos que precisan una especial revisión, destacan los relacionados con la protección de los proveedores de servicios.

${ }^{4}$ RODRIGUEZ-PIÑERO ROYO, Miguel. «El trabajo 3.0 y la regulación laboral: por un enfoque creativo en su tratamiento legal». Departamento de Derecho del Trabajo de la Universidad de Sevilla. Universidad de Sevilla. http://grupo.us.es/iwpr/2016/09/07/eltrabajo-3-0-y-la-regulacion-laboral-por-un-enfoque-creativo-en-su-tratamiento-legal/ (Última consulta 12 de abril de 2017).

${ }^{5}$ COMITÉ DE LAS REGIONES. Dictamen sobre la dimensión local y regional de la economía colaborativa. Bruselas: Comité de las Regiones de la Unión Europea, 2015.

${ }^{6}$ O.I.T. Recomendación $n .^{\circ} 204$ sobre transición de la economía informal a la formal. Ginebra: Organización Internacional del Trabajo, 2015. 
En este sentido, la institución europea recoge que «las empresas con ánimo de lucro a veces utilizan de forma abusiva las plataformas de la «gig-economy» y no proporcionan a sus trabajadores la protección y cobertura laboral necesaria» ${ }^{7}$. Dicho esto, conviene cuestionarse si estos «microtrabajadores» constituyen o no una nueva manifestación del trabajo precario.

En palabras de AJA VALLE ${ }^{8}$, la precariedad se asocia como «una vulnerabilidad e inseguridad laboral que, yendo más allá del tipo de contrato, condiciona la posibilidad real de emancipación y desarrollo personal de los trabajadores». Así, en el universo de «gig-economy», la O.I.T. ha identificado tres principales áreas de potencial inseguridad que podrían tildar de precario el trabajo que se desarrolla en las plataformas.

En relación con el empleo, se destaca la preocupación derivada de la inestabilidad económica. En este sentido, las ganancias de los trabajadores de las plataformas de la «gig-economy» no solo se encuentran profundamente sujetas a las fluctuaciones del mercado de la demanda, sino que la demanda entra en un mercado en el que el lado de la oferta está también sujeto a olas altamente variables que dependen de las condiciones generales del mercado de trabajo. Así, la incertidumbre por la falta de continuidad en la prestación del servicio o por la rebaja de los ingresos pesa en el día a día de los trabajadores. Además, en ocasiones, sus ingresos se pueden ver alterados por decisiones unilaterales de las plataformas. En este sentido, cabe destacar el caso de Uber, en el que la compañía puede hacer oscilar de forma unilateral los precios de los viajes de transporte ofertados entre un 20 y un $30 \%$, repercutiendo sobre los ingresos del conductor.

En relación con los horarios, estas plataformas virtuales no ejercen ningún tipo de control sobre las horas que pueden trabajar los proveedores de servicios. En este sentido, cabe destacar que la limitación del tiempo de trabajo, objeto de atención legislativa desde los inicios de la legislación social y también posteriormente en el desarrollo del Derecho del Trabajo, se ha configurado tradicionalmente como una tutela de la salud y seguridad de los trabajadores. Así, en estos casos, la inexistencia de límites temporales en la prestación de los servicios expone a los trabajadores a un fuerte riesgo de agotamiento, con repercusiones en su bienestar psicofísico y en la calidad del servicio que ofrece. De hecho, no resulta extraño que, a resultas de la inestabilidad de las ganancias, aquellos que mayores dificultades soportan, entren en el círculo vicioso de aceptar las peores ofertas, reduciéndose sus ingresos y teniendo que trabajar más horas para cubrir los costes de su actividad.

${ }^{7}$ COMITÉ DE LAS REGIONES. Dictamen sobre la dimensión local y regional de la economía colaborativa. Bruselas: Comité de las Regiones de la Unión Europea, 2015.

${ }^{8}$ LACALLE, Daniel. Trabajadores precarios, trabajadores sin derechos. Primera edición. Madrid: Ed. El Viejo Topo, 2009, pp. 12-16. 
Además, esta falta de control por parte de las plataformas virtuales choca con la regulación que existe en algunos sectores específicos sobre esta materia. Por ejemplo, en el sector del transporte por carretera de personas o mercancías, aplicable al modelo de Uber, tanto el regulador comunitario como el estatal han intervenido para reforzar la seguridad y salud de los transportistas. Así, el Real Decreto de 2013, sobre ordenación del tiempo de trabajo para los trabajadores autónomos que realizan actividades móviles de transporte por carretera, impuso unas cotas máximas al tiempo de trabajo9.

Por último, en lo relativo a los derechos de representación, la gig economy, al quedar fundamentada sobre una clase trabajadora no agrupada y casi invisible, no se beneficia de un poder sindical fuerte que luche por unas condiciones de trabajo más justas ${ }^{10}$. Esto incide tanto en los medios de acción colectiva, como en las tutelas que las acompañan. Así, en relación con el derecho de huelga, si desde el punto de vista sustancial es difícil organizar una protesta efectiva y eficaz en forma de abstención de trabajo, desde el punto de vista normativo, tal abstención no parecería revestir las características de la huelga y, en función de las acciones que se deberían adoptar, también podría dar lugar a represalias por parte de las plataformas. Lo mismo sucede con los derechos vinculados a la contratación colectiva y a las prerrogativas de los representantes de los trabajadores, de fundamental importancia para la explicación de una acción colectiva de relevancia ${ }^{11}$.

Por tanto, parece que la ausencia de regulación deja a los proveedores de estas plataformas en un limbo legal caracterizado por la ausencia de garantías. Así, la descentralización productiva y el recurso al trabajo autónomo como fórmula principal para la prestación de servicios parece camuflar una huida del Derecho del Trabajo, con la consiguiente movilización de los trabajadores hacia empleos próximos a la precariedad.

9 Artículo 3 del RD 128/20130: «La duración media del tiempo de trabajo semanal no debe sobrepasar las 48 horas. No obstante, podrá prolongar el tiempo de trabajo hasta 60 horas siempre que la duración media del mismo no supere las 48 a la semana en un periodo de cuatro meses naturales».

${ }_{10}$ Precisamente, sobre este punto, sindicatos de Alemania, Austria, Suecia, Dinamarca y Estados Unidos han suscrito recientemente el conocido como «Documento Frankfurt», relativo al trabajo basado en plataformas, en el que reclaman el derecho a organizarse para los trabajadores de plataformas digitales e incluso a participar en la dirección de las mismas. En esta misma línea, la Confederación Europea de Sindicatos también ha manifestado su preocupación por la extensión del trabajo digital precario y la necesidad de reforzar la acción sindical en estas nuevas formas de organización no convencionales.

11 DAGNINO, Emanuele, «Uber law: perspectiva jurídico-laboral de la sharing/ondemand economy». Revista Internacional y Comparada de Relaciones Laborales y Derecho del Empleo. 2015, Vol. III, n. ${ }^{\circ}$ 3, pp. 1-32. 


\section{Relación entre la "gig economy» y las E.T.T's}

Siguiendo la definición propuesta en la Ley 14/1994 ${ }^{12}$, las Empresas de Trabajo Temporal (en adelante, ETT's) son empresas privadas cuya actividad principal consiste en satisfacer las necesidades temporales y urgentes de mano de obra de otros empresarios, mediante la cesión temporal a los mismos de trabajadores.

Así, las ETT's ofrecen un control de calidad de los trabajadores temporales para que no lo tengan que hacer las empresas. Un control de calidad que se basa en tres actuaciones: por un lado, se encargan de la selección de los trabajadores (control de entrada), en segundo lugar, son responsables de su formación (control de resultados), librando a la empresa receptora de ejecutar esa labor y, por último, tal y como recoge el artículo 15.2 de la Ley 14/1994, ejercen el poder disciplinario sobre el trabajador en cuestión (control de salida). Respecto a este último punto, las ETT's se aseguran de que, si un trabajador no cumple, será despedido por la ETT y no será enviado de nuevo a las empresas que contraten con esa ETT.

Las plataformas de la «gig-economy», por su parte, han logrado a través de sus sistemas reputacionales desempeñar estas mismas funciones de una forma más barata y constituyen una amenaza real para las tradicionales ETT's. Esto es, un conductor de Uber que tenga una puntuación de cinco estrellas, asegura al cliente que ese conductor es de «calidad» (control de entrada). A su vez, los conductores que tengan bajas puntuaciones serán «desactivados» por la plataforma impidiendo que se realicen negocios con ellos a través de ésta (control de salida). Por último, si los conductores desean obtener buenas puntuaciones necesitan estar formados y preparados para el trabajo. En ese sentido, Uber no necesita formarlos ya que el sistema crea los incentivos suficientes (transparencia del mercado y amenaza de despido) para que los trabajadores tengan que formarse por ellos mismos si quieren ofrecer sus servicios.

Al hilo de lo anterior, conviene señalar que, aun realizando prácticamente las mismas funciones que las ETT's, las plataformas virtuales de la «gig-economy» no se hallan sujetas a ningún requisito ni regulación para el desarrollo de su actividad. Así, si bien la Ley 14/1994, reguladora de las ETT's, propone una serie de cautelas para prevenir la aparición de intermediarios en el mercado de trabajo capaces de eludir sus obligaciones laborales y de seguridad social, sorprende que sean precisamente las plataformas digitales, en las que prolifera el empleo precario, las que no deban cumplir

12 Artículo 1 de la Ley 14/1994, de 1 de junio, por la que se regulan las empresas de trabajo temporal. 
con ninguna restricción. En este sentido, se posiciona TODOLÍ SIGNES ${ }^{13}$ quien sostiene que la intermediación a través de empresas de la «gig-economy» sustituirá el rol que desempañan las ETT's si el regulador no actúa con la suficiente rapidez.

\section{Uber como manifestación de la «gig-economy» en España}

Uber se posiciona como una de las compañías más representativas de la «gig-economy» en el mundo. Así, el servicio de transporte que ofrece se desarrolla de la siguiente forma: el usuario descarga de forma gratuita la aplicación de Uber en su dispositivo móvil, y ésta, mediante un sistema GPS, localiza los vehículos disponibles más cercanos. Una vez escogido uno de los vehículos y especificado el destino, la aplicación facilita al usuario una estimación tanto del coste como de la duración del recorrido. Finalizado el servicio, se procede al pago a través de la aplicación móvil sin que quepa negociación entre ninguna de las dos partes sobre el precio del servicio.

Uberpop, filial de Uber, desembarcó por primera vez en España en abril de 2014. Sin embargo, a finales de ese mismo año ya contaba con dos demandas reclamando el cese de su actividad, alegando, entre otras causas, supuestos actos de competencia desleal. La primera, interpuesta contra el Juzgado Mercantil n. ${ }^{0} 2$ de Madrid, dio lugar a la suspensión ${ }^{14}$, de forma cautelar, de la actividad de Uberpop en España. La segunda, interpuesta contra el Juzgado Mercantil n. ${ }^{\circ} 3$ de Barcelona, propició que el Juzgado presentara una cuestión prejudicial ${ }^{15}$ ante el Tribunal de Justicia de la Unión Europea ${ }^{16}$. Esta misma decisión también acabó tomando el Juzgado de Madrid, decidiendo en la vista previa del juicio suspenderlo y elevar la consulta al Tribunal de Luxemburgo.

Con independencia de estos procesos, que versan principalmente sobre materias mercantiles - pero que también podrían llegar a resultar de utilidad para determinar la naturaleza jurídica del servicio ofrecido por Uberpop-, también están surgiendo dudas sobre las implicaciones laborales de estas fórmulas colaborativas. Así, si bien el modo de prestación de servicios en estas experiencias reúne particularidades que las apartan claramente de las formas laborales tradicionales, es innegable que persisten en algunos casos factores

${ }^{13}$ TODOLÍ SIGNES, Adrián. El trabajo de la era economía colaborativa. Primera edición. Valencia: Ed. Tirant lo Blanch, 2017, p. 147.

${ }_{14}$ Auto de medidas cautelares del Juzgado Mercantil n. ${ }^{\circ} 2$ de Madrid, de 9 de diciembre de 2014, n. $^{\text {7 } 707 / 2014 . ~}$

${ }_{16}$ Providencia del Juzgado Mercantil n. 3 de Barcelona, de 17 de junio de 2015, relativa al asunto n. ${ }^{\circ}$ 929/2014D2 dando traslado de una cuestión prejudicial ante el TJUE. 
que pueden hacer dudar de la presencia de una relación laboral subyacente (restricciones, recepción de instrucciones, fijación de precios por hora de trabajo...).

En este sentido, están surgiendo tanto en el ámbito nacional como internacional resoluciones que urgen a la necesidad de analizar profundamente las relaciones laborales que se dan entre Uber y sus conductores. En España, la Inspección de Trabajo y de la Seguridad Social de Cataluña ya se ha pronunciado, sin consecuencias definitivas aún, a la espera de que los Tribunales Europeos determinen si la actividad de la empresa es o no legal. Dicho esto, el debate de si la relación es laboral -por cuenta ajena- o autónoma -por cuenta propia-, también está planteándose en otros lugares del mundo. Así, recientemente, el Tribunal Central de Trabajo de Londres, en sentencia de 28 de octubre de 2016, también se pronunció sobre este extremo ${ }^{17}$. Y, con carácter previo, la Comisión Laboral de California, en junio de $2015^{18}$, ya analizó esta misma problemática.

En todas las citadas resoluciones, los análisis conducen a afirmar la existencia de una relación de laboralidad entre la empresa Uber y sus conductores. De esta forma, se valorará en lo sucesivo los argumentos que han llevado a estas conclusiones, los problemas que genera su encaje en esta categoría y las posibles soluciones jurídicas que cabe plantear para quienes presten sus servicios a través este tipo de plataformas digitales.

\section{SOBRE LA LABORALIDAD EN LAS RELACIONES ENTRE UBER Y SUS CONDUCTORES}

El surgimiento de esta nueva forma de interacción entre plataformas y usuarios exige necesariamente una correcta delimitación del campo de aplicación subjetivo del ordenamiento laboral. Así, como la doctrina ha puesto de manifiesto en más de una ocasión, el dinamismo del sistema productivo y ocupacional, así como la propia mentalidad social, irremediablemente provocan constantes replanteamientos sobre quiénes deben ser considerados trabajadores por cuenta ajena y, por tanto, tutelados por el ordenamiento laboral, $\mathrm{y}$, quienes han de ser catalogados como trabajadores por cuenta propia. Obviamente, las repercusiones de la inserción en una u otra categoría son importantes tanto desde el punto de vista del empresario (encarecimiento de

17 Sentencia del Tribunal Central de Trabajo de Londres, de 28 de octubre de 2016 n. ${ }^{\circ}$ 2202551/2015, https://www.judiciary.gov.uk/judgments/mr-y-aslam-mr-j-farrar-andothers-v-uber/ (Última consulta el 12 de abril de 2017).

${ }^{18}$ Sentencia del Tribunal Superior de California, de 28 de octubre de 2016 n. ${ }^{\circ}$ CGC15-546378. https://es.scribd.com/doc/268911290/Uber-vs-Berwick (Última consulta el 12 de abril de 2017). 
los costes fijos laborales) como desde la perspectiva del trabajador (protección laboral).

Dicho esto, para poder confirmar o excluir la existencia de una relación laboral entre la empresa y los conductores resulta indispensable recordar previamente las notas características de la relación laboral. Así, una vez analizadas éstas y aplicado el test de laboralidad a los conductores de Uber, se estudiarán los hasta ahora existentes pronunciamientos administrativos, jurisprudenciales y doctrinales sobre esta materia. Por último, se valorarán los principales problemas que genera el encaje de este nuevo tipo de trabajadores en el concepto tradicional del contrato de trabajo.

\section{Concepto tradicional del trabajador por cuenta ajena}

\subsection{Elementos definitorios de la relación laboral y su distinción frente al trabajo autónomo}

El artículo 1.1 del Estatuto de los Trabajadores delimita el ámbito de aplicación de la normativa laboral, estableciendo que ésta alcanza a quienes «voluntariamente presten sus servicios retribuidos por cuenta ajena y dentro del ámbito de organización y dirección de otra persona, física o jurídica, denominada empleador o empresario». De esta definición se desprenden las cuatro notas que caracterizan la relación laboral: voluntariedad, ajenidad, subordinación o dependencia funcional y retribución. Por su parte, el trabajo autónomo, regulado en el artículo 1.1 de la Ley 20/2007, de 11 de Julio, del Estatuto del Trabajador Autónomo, se define como el realizado por «personas físicas que realicen de forma habitual, personal, directa, por cuenta propia y fuera del ámbito de dirección y organización de otra persona, una actividad económica o profesional a título lucrativo, den o no ocupación a trabajador por cuenta ajena.»

En la comparación entre ambas definiciones, cabe interesarse por las notas de ajenidad y subordinación o dependencia funcional por ser aquellas características que, como recoge la jurisprudencia, constituyen los dos elementos clave para diferenciar el trabajo por cuenta ajena del trabajo por cuenta propia. Así, a modo ilustrativo, cabe destacar la sentencia del Tribunal Supremo de 7 de octubre de $2009^{19}$, que establece:

"[mientras] en el contrato de arrendamiento de servicios el esquema de la relación contractual es un genérico intercambio de obligaciones y prestaciones de trabajo con la contrapartida de un precio o remuneración de los servicios; en el contrato de trabajo dicho esquema o causa objetiva del tipo contractual es una especie del género anterior que consiste en el in-

19 STS de 7 de octubre de 2009, n. ${ }^{\circ} 6415 / 2009$, F.j. 2. 
tercambio de obligaciones y prestaciones de trabajo dependiente por cuenta ajena a cambio de retribución garantizada; cuando concurren, junto a las notas genéricas de trabajo y retribución, las notas especificas de ajenidad del trabajo y de dependencia en el régimen de ejecución del mismo nos encontramos ante un contrato de trabajo, sometido a la legislación laboral.»

Así, la nota de ajenidad ha sido tradicionalmente explicada desde dos perspectivas: por un lado, como transmisión automática de los frutos del trabajador a quien recibe la prestación del servicio, «ajenidad en los frutos»; $\mathrm{y}$, por otro lado, como atribución a esa misma persona de los riesgos económicos o de explotación propios de la actividad de que se trate, «ajenidad en los riesgos». Sin embargo, cabe añadir una tercera perspectiva, la «ajenidad en el mercado $»{ }^{20}$, que alude a la desconexión entre el trabajador y el destinatario final del producto de su trabajo, por la interposición de un tercero: el empresario. Paralelamente, la nota de subordinación o dependencia funcional hace referencia a la realización del trabajo bajo las órdenes e instrucciones de otra persona, dentro de su ámbito de organización y dirección.

Por tanto, en relación con lo anterior, cabe inferir que mientras los trabajadores autónomos prestan sus servicios con independencia y autonomía de criterio, asumiendo el riesgo, véase los beneficios y costes, de la actividad económica, los trabajadores por cuenta ajena prestan sus servicios dentro del ámbito de dirección, organización y control de otro sujeto sin asumir el riesgo y ventura de la actividad empresarial.

\subsection{Flexibilización de la nota de subordinación y dependencia funcional}

Si bien en una primera fase la nota de subordinación y dependencia funcional fue entendida por los tribunales como sujeción estricta a las órdenes de otra persona, las nuevas tecnologías y los procesos de descentralización productiva sufridos en el mercado laboral han flexibilizado notablemente su interpretación. Con el paso del tiempo, esta nota se ha contemplado más bien como una inserción del trabajador en un círculo de dirección y programación ajena. Así, a día de hoy, la protección laboral también alcanza a personas que trabajan previo encargo del cliente o libertad de horario, siempre que desarrollen su trabajo dentro del ámbito de organización del empresario. En este sentido, la jurisprudencia viene estableciendo que el trabajo subordinado no es incompatible con un -incluso amplio- espacio de autonomía en el desarrollo de la actividad profesional, tal y como recoge la sentencia del Tribunal

20 ALARCÓN CARACUEL, Manuel Ramón. «La ajenidad en el mercado: un criterio definitorio del contrato de trabajo». Revista Española de Derecho del Trabajo, 1986, n. ${ }^{\circ} 28$, pp. $495-544$. 
Supremo de 19 de febrero de $2014^{21}$ : «la dependencia, entendida como esa integración «en el ámbito de organización y dirección del empresario», resulta la cristalización de una larga elaboración jurisprudencial en la que se concluyó que no se opone a que concurra esta nota de la dependencia la «autonomía profesional» imprescindible en determinadas actividades.»

Del mismo modo, también cabe apreciar el impacto que la digitalización está teniendo sobre el control del tiempo por parte del empresario. Así, las especiales características de las innovaciones tecnológicas han dado pie a la sustitución del clásico control periférico, discontinuo y parcial por sistemas digitales centralizados. Esto es, donde antes reinaba el viejo taylorismo, en el que el capataz debía manejar y presionar a sus obreros a fin de mantener ágiles los ritmos de producción, hoy se desarrollan nuevos sistemas de control tecnológico que ejercen el control sobre la propia maquinaria, a través de softwares que identifican al trabajador y el tiempo de trabajo que efectivamente desarrollan ${ }^{21}$.

\section{Aplicación del test de laboralidad a los trabajadores de UBER}

En Uber, los conductores, denominados partners, tienen la consideración de trabajadores en tanto que, además de ser titulares del vehículo y asumir directamente los gastos de actividad, deciden libremente cuanto y cuando trabajar, sin quedar sujetos a una jornada mínima o a unos horarios predeterminados. Precisamente, esta libertad organizativa de la que gozan los conductores de la plataforma es utilizada por la empresa para declarar su naturaleza de mera empresa tecnológica, cuya actividad consiste únicamente en disponer los medios técnicos y organizativos necesarios para facilitar el contacto entre usuarios y titulares de vehículos para desplazamientos urbanos ${ }^{22}$.

Sin embargo, en la realidad, Uber cobra un mayor protagonismo del que dice tener. Así, es sobradamente conocido que interviene en la prestación del servicio puesto que lleva a cabo acciones que van desde la implementación de decisiones organizativas (fijación de los precios) hasta el control de la actividad de sus conductores (procesos de selección). Por tanto, desde la perspectiva del Derecho del Trabajo, resulta necesario abordar si esta intervención puede tildar de laboral la relación entre el conductor y la plataforma tecnológica. Así, independientemente del «nomen iuris» que errónea

${ }^{21}$ STS de 19 de febrero de 2014, n. ${ }^{\circ}$ 1404/2014, F.j. 2.

${ }^{22}$ GINES I FABRELLAS, Anna.; GALVEZ DURÁN, Sergi. «Sharing economy vs. Uber economy y las fronteras del Derecho del Trabajo: la (des)protección de los trabjadores en el nuevo entorno digital.» Revista para el análisis del Derecho, 2016, n. ${ }^{\circ}$ 1/2016, pp. $12-16$. 
o interesadamente pueda establecer la plataforma, tal y como recoge la jurisprudencia del Tribunal Supremo ${ }^{23}$, «los contratos tienen la naturaleza que se deriva de su real contenido obligacional, la que se derive de los requisitos que determinan la laboralidad y de las prestaciones realmente llevadas a cabo». Por tanto, se analiza en lo sucesivo la concurrencia de las notas características del artículo 1.1. del Estatuto de los Trabajadores, poniendo especial atención en las notas de ajenidad y subordinación o dependencia funcional.

\subsection{Voluntariedad}

La concurrencia de voluntades entre quien ofrece y demanda empleo, sin perjuicio de que pueda concertarse de forma expresa o tácita, constituye un elemento esencial para la calificación del trabajador como asalariado. En este caso, existe voluntariedad en la prestación de los servicios en el sentido en el que nadie obliga a los conductores a efectuar su trabajo, es más, tienen incluso la posibilidad de rechazar determinados viajes.

\subsection{Retribución}

En cuanto a la retribución, cabe destacar que los conductores de la plataforma virtual reciben una contraprestación por el servicio realizado y no directamente por el usuario o cliente. Este dato, que en principio puede resultar insignificante, es determinante, ya que los clientes pagan directamente a través de Uber por el servicio de los conductores y, sólo posteriormente, la propia plataforma hace entrega de la parte proporcional de la tarifa al conductor, gestionando de este modo su remuneración ${ }^{24}$.

Ciertamente, la forma de contraprestación ha sido tradicionalmente considerada como un buen indicio para distinguir entre trabajo asalariado y el que no lo es. Así, la retribución fija y periódica es indicio de trabajo asalariado, mientras que la retribución por acto o servicio o la compensación en función de los beneficios de la sociedad han sido considerados como indicios de trabajo por cuenta propia o trabajo no dependiente. Ahora bien, el simple hecho de cobrar por pieza, acto o unidad de trabajo, como resulta en el caso de Uber, no es suficiente para negar por sí solo el carácter laboral del vínculo puesto que lo decisivo, tal y como recoge la sentencia del Tribunal Supremo

${ }^{23}$ STS de 20 de marzo de 2007, n. ${ }^{\text {o } 2546 / 2007, \text { F.j. } 3 .}$

24 ARAGÜEZ VALENZUELA, Lucía. «Nuevos modelos de economía compartida: Uber economy como plataforma virtual de prestación de servicios y su impacto en las relaciones laborales.» Revista Internacional y Comparada de Relaciones Laborales y Derecho del Empleo, 2017, Vol. V, n. ${ }^{\circ}$ 1, pp. 167-190. 
de 29 de diciembre de $1999^{25}$, es que el trabajo se preste en condiciones de ajenidad y dependencia:

«el no establecimiento de retribución o salario fijo, a pesar de lo afirmado en la sentencia recurrida, no es un elemento característico delimitador del contrato de trabajo respecto de otras figuras, dado el concepto de salario contenido en el art. 26.1 ET comprensivo de «la totalidad de las percepciones económicas de los trabajadores, en dinero o en especie, por la prestación profesional de los servicios laborales por cuenta ajena, ya retribuyan el trabajo efectivo, cualquiera que sea la forma de remuneración, o los periodos de descanso computables como de trabajo».»

Por tanto, conviene analizar en detalle esas dos notas características.

\subsection{Subordinación o dependencia funcional}

Tal y como ha quedado acreditado previamente, la interpretación de la nota de subordinación o dependencia funcional viene sufriendo una constante modulación en los últimos años. Así, a día de hoy, esta característica no se restringe únicamente a la existencia de instrucciones precisas sobre la forma de prestación del servicio, sino más bien a la integración de los trabajadores en la organización empresarial. De esta forma, ha de valorarse si los conductores de Uber quedan sujetos a los poderes de dirección, organización y control de la empresa.

Para ello, haciendo uso de la técnica indiciaria que aconseja la sentencia del Tribunal Supremo de 20 de enero de $2015^{26}$ «La calificación de la relación como laboral ha de hacerse en cada caso en atención a los indicios existentes, valorando principalmente el margen de autonomía del que goza quien presta el servicio.», resaltaremos tres principales manifestaciones que podrían apoyar la existencia de subordinación y dependencia en el caso de Uber.

a) Establecimiento de concretas instrucciones e indicaciones sobre la forma de prestación de servicios

En el caso de Uber, la plataforma dicta instrucciones (directas) o recomendaciones (indirectas) a los proveedores de servicios sobre cómo realizar la prestación del servicio. Así, a modo de ejemplo, conviene señalar los consejos que recibieron los conductores de Uber Barcelona durante el tiempo en que la plataforma estaba legalizada: se recomendaba llegar a la dirección indicada rápidamente, mostrar una actitud agradable y distendida o mantener

\footnotetext{
${ }^{25}$ STS de 29 de diciembre de 1999, n. $^{\circ} 8532 / 1999$, F.j. 2.

${ }^{26}$ STS de 20 de enero de 2015, n. ${ }^{\circ}$ 637/2015, F.j. 2.
} 
el coche limpio y en buenas condiciones. Respecto a las instrucciones directas, cabe destacar, entre otros ejemplos, que Uber es quien establece la ruta a seguir por sus conductores o que, una vez aceptado el viaje por el conductor, éste ya no puedo rechazarlo.

b) Toma de decisiones estratégicas y comerciales por parte de la empresa.

La fijación de los precios de los trayectos queda establecida unilateralmente por la plataforma quedando bajo su poder de decisión tanto los cambios en la política tarifaria como las modificaciones que desee introducir respecto de la proporción que los conductores deben abonar a Uber. En este sentido, cabe recordar lo recogido por la sentencia de 23 de noviembre de $2009^{27}$, que indica que «la adopción por parte del empresario y no del trabajador de las decisiones concernientes a las relaciones de mercado o de las relaciones con el público, como fijación de precios o tarifas, selección de clientela, indicación de personas a atender» evidencia la presencia de relación laboral.

c) Los conductores se hallan sujetos a un permanente sistema de control.

Por último, otro de los indicios que debe ser resaltado se asocia con la capacidad de la plataforma para «desactivar» a los conductores por razones de rendimiento o disciplina. Así, una de las herramientas con la que cuenta la plataforma para acometer este control es el sistema de evaluación por parte de los clientes. De esta forma, en el caso de obtener puntuaciones negativas, se procede a dar de baja el perfil del conductor y, en caso de no llegar al umbral del 4,5/5 en el conjunto de sus 25 primeros viajes, los prestadores del servicio no pueden acceder al sistema de incentivos que ofrece la compañía. En este sentido, cabe destacar la doctrina del Tribunal Supremo, la cual asume que los sistemas de control de la actividad productiva que incluyen valoraciones de los clientes suponen un indicio de laboralidad. En este sentido, se pronuncia la ya mencionada sentencia de 29 de diciembre de $1999^{28}$, que establece: «la empresa cuenta con un servicio de inspección que revisaba, entre otros, el trabajo realizado por el actor y recibe las quejas que pudieran tener los clientes sobre su actividad».

Unido a esto, y en consonancia con lo antes comentado, el control que ejerce en estos casos la plataforma se ejecuta a través de cauces informáticos. Así, el denominado «software in accounting» instalado en los dispositivos móviles de los conductores de Uber se vincula con la empresa, que es la que recibe automáticamente no sólo los tiempos de trabajo sino la frecuencia de los servicios o de las interrupciones y su duración.

27 STS de 23 de noviembre de 2009, n. ${ }^{\circ} 8549 / 2009$, F.j. 2.

${ }^{28}$ STS de 29 de diciembre de 1999, n. ${ }^{\circ} 8532 / 1999$, F.j. 2. 


\subsection{Ajenidad}

Como ya ha sido adelantado, la nota de la ajenidad se explica a través de tres dimensiones, la ajenidad en los frutos, en los riesgos y en el mercado. Así, en base a estas tres vertientes, valoraremos si concurre o no esta característica en el caso de Uber.

\section{a) Ajenidad en los frutos}

La ajenidad en los frutos implica que los resultados del trabajo son atribuidos inicial y directamente a persona distinta de la que ejecuta el trabajo, una atribución originaria que es previa a la ejecución del trabajo ${ }^{29}$. En el caso de Uber, el sistema existente permite que la retribución sea abonada por parte de los clientes a la compañía, cargándose automáticamente el importe a la tarjeta de crédito, sin que exista intervención del conductor. Así, este último, ingresa el dinero con carácter posterior y una vez que resulta detraída la comisión por parte de Uber. Dicho esto, parece existir apropiación por parte de Uber de los resultados de la actividad productiva de sus conductores ya que el pago se realiza a la empresa y no directamente al prestador del servicio. En este mismo sentido, se han pronunciado los Tribunales Españoles, tal y como recoge la sentencia del Tribunal Supremo, de 6 de octubre de $2010^{30}$ :

«En efecto, concurren en el caso todos los rasgos definitorios de la laboralidad establecidos en el artículo 1 del ET y desarrollados por la jurisprudencia a lo largo de años de actividad interpretativa, [...] la utilidad patrimonial derivada del mismo - es decir, lo que pagan los clientes-ingresa directamente en el patrimonio de la empresa y no en el de los actores (ajenidad en los frutos y en la utilidad patrimonial) y estos percibirán su salario, en la modalidad de por unidad de obra.»

b) Ajenidad en los riesgos

Ciertamente, los conductores de Uber soportan mayores riesgos que los propios de una relación laboral convencional. En este sentido, Uber alega que además de que los conductores han de utilizar sus propios medios de producción, deben también sufragar los gastos derivados de la actividad. Sumado a esto, la plataforma defiende igualmente que el sistema de retribución comporta un riesgo añadido al hacer depender los ingresos del número de trayectos realizados.

Sin embargo, en relación con esto último, cabe destacar que aunque la retribución por servicio comporte la asunción de cierto riesgo por depender

${ }^{29}$ OJEDA AVILÉS, Antonio. «Ajenidad, dependencia o control: la causa del contrato.» Tribuna Social: Revista de Seguridad Social y Laboral, 2007, n. ${ }^{\circ} 195$, pp. 13-24.

${ }^{30}$ STS de 6 de octubre de 2010, n. ${ }^{\circ}$ 5384/2010, F.j. 3. 
la retribución de la efectiva prestación del servicio, no puede afirmarse que exista asunción del riesgo de la actividad productiva por parte del conductor. De hecho, así lo ha manifestado el Tribunal Supremo, en sentencia de 10 de Julio de $2007^{31}$, «resulta un claro indicio de ajenidad [...] el cálculo de la retribución o de los principales conceptos de la misma con arreglo a un criterio que guarde una cierta proporción con la actividad prestada, sin el riesgo y sin el lucro especial que caracterizan a la actividad del empresario o al ejercicio libre de las profesiones».

Por otro lado, en lo referente a los medios productivos aportados por el trabajador, cabe destacar que éstos tienen escasa entidad -tanto desde un punto de vista cuantitativo como cualitativo- en comparación con los verdaderos medios de producción de la actividad económica, como son la propia plataforma virtual, la aplicación móvil y los medios humanos específicos empleados por Uber para la oferta del servicio de transporte de viajeros.

Por lo tanto, si bien se asume un mayor grado de riesgo, este no parece quedar asociado con la asunción del riesgo y ventura de la actividad económica. En este sentido, los conductores, más allá de aportar el vehículo y su propia fuerza de trabajo, no intervienen en el negocio de Uber, por cuanto el trabajo que realizan no comporta una actividad de organización empresarial que pueda afectar a los resultados del negocio.

\section{c) Ajenidad en el mercado}

La ajenidad en el mercado significa que entre el trabajador directo, que produce un bien o presta un servicio, y el consumidor, se interpone jurídicamente un tercero (el empresario), que, cobrando un precio por ese bien o servicio, pagará un salario al trabajador y tendrá la posibilidad de obtener un beneficio.

En el caso de Uber, resulta evidente que entre el conductor y el usuario intermedia la plataforma puesto que quien cobra por el servicio, como ya se ha adelantado, no es el conductor sino la propia plataforma, que posteriormente le remunera. En este sentido, vuelve a ser de interés la sentencia citada al hablar de la ajenidad en los frutos, que establece lo siguiente: «Los clientes (productoras de cine y televisión) contratan con la empresa y no con los actores la realización de los doblajes (ajenidad en el mercado)», supuesto extrapolable al caso de los clientes de Uber ya que no contratan con los propios conductores sino con la plataforma.

\section{d) Conclusión}

Sentado el análisis, parece apreciarse en la relación entre Uber y sus conductores los cuatro rasgos definitorios de la laboralidad, lo que llevaría a la

${ }^{31}$ STS de 10 de julio de 2007, n. ${ }^{\text { 5758/2007, F.j. } 3 .}$ 
inevitable aplicación de la normativa laboral. Así, como refuerzo a esta conclusión, se examinan en lo sucesivo los principales pronunciamientos administrativos, jurisprudenciales y doctrinales sobre esta materia.

\section{Pronunciamientos administrativos, jurisprudenciales y doctrinales}

\subsection{Administrativos: Informe de la Inspección de Trabajo y Seguridad Social Catalana ${ }^{32}$}

Tras siete meses de investigación, la Inspección de Trabajo y Seguridad Social de Cataluña publicó, en marzo de 2015, un informe en el que concluía que la relación jurídica que une a los conductores con Uber no podía ser calificada de arrendamiento de servicios, sino de relación laboral. Para sostener esta postura, la Inspección afirmó que concurrían los elementos constitutivos de dependencia y ajenidad propios del contrato de trabajo.

Así, en relación con la dependencia, el informe recogía que los conductores recibían directrices internas de trabajo mediante mensajes de correo electrónico o móvil del empleado de Uber que les era asignado como contacto. Asimismo, también se especificaban otros indicios que evidenciaban una clara conducta organizativa por parte de Uber, entre los que cabe destacar, la creación de un sistema de solución de conflictos con los conductores, la creación de un sistema de atención al cliente (servicio centralizado de objetos perdidos), la fijación de las tarifas del servicio o la facilitación de un smartphone con el software necesario instalado. Además, a este poder de dirección y organización, también se sumaban otras acciones que evidenciaban el poder de control por parte de Uber frente a sus conductores. En este sentido, destaca la Inspección que es la empresa quien sanciona el rechazo de clientes, controla su conexión y su desconexión al sistema operativo y puede dar de baja a los conductores en caso de malas valoraciones, ya sea por parte de los clientes o por el rechazo consecutivo de viajes.

Por otro lado, en lo referente a la ajenidad, la Inspección justificó este rasgo vinculándolo con la idea de que, a pesar de que el conductor de Uber asuma los riesgos por la prestación del servicio (por ejemplo, los daños ocasionados en caso de accidente) e incluso los frutos ocasionados por su trabajo (como puede entenderse el mantener correctamente el vehículo, asumir las sanciones por el transporte, etc.), esto debe interpretarse en el sentido de que los riesgos se derivan de la rentabilidad de la empresa. Con ello, el conductor

${ }^{32}$ GOZZER, Stefania. «Trabajo dice que los chóferes de Uber son empleados de la firma». El País. 13 de junio de 2015. 
recibe una contraprestación económica por el servicio prestado que no fluctúa en beneficio o perjuicio de su servicio ${ }^{33}$.

De esta forma, la Inspección reivindica la laboralidad de esta relación pero se mantiene a la espera del pronunciamiento de los Tribunales sobre la legalidad del servicio para proponer las correspondientes sanciones.

\subsection{Jurisprudenciales: Sentencia del Tribunal Central de Trabajo de} Londres y dictamen de la Comisión de Trabajo de California.

a) Sentencia del Tribunal Central de Trabajo de Londres ${ }^{34}$

El Tribunal Central de Trabajo de Londres (en adelante, TCT) dictó sentencia el 28 de octubre de 2016, en la que dirime la disputa entre Uber y un colectivo de sus conductores británicos, radicados en el área metropolitana de Londres, que reclamaban ser considerados trabajadores por cuenta ajena de la compañía, al entender que la consideración de trabajadores autónomos era contraria a la ley laboral inglesa.

El TCT realiza nuevamente el tradicional test de laboralidad, sin embargo, en este caso, además de incluir los indicios «clásicos» de laboralidad (que se corresponden con los que enumera el Informe de la Inspección de Trabaja y Seguridad Social de Cataluña), introduce cinco nuevos indicios, cuyo estudio resulta interesante ${ }^{35}$ :

En primer lugar, la sentencia considera indicio de laboralidad la imposibilidad que tienen estos autónomos de crecimiento. En este sentido, si efectivamente fueran pequeños empresarios lo lógico sería que existieran unas mínimas posibilidades de crecimiento en el negocio. Así, como establece la sentencia, las únicas posibilidades de crecimiento de los conductores se reducen a trabajar más horas. Es decir, estos conductores realmente solo aportan mano de obra, por lo que no pueden considerarse empresarios.

Por otro lado, también se presenta como un indicio de laboralidad el que los conductores conduzcan bajo una marca ajena. Asimismo, la sentencia

33 ARAGÜEZ VALENZUELA, Lucía. «Nuevos modelos de economía compartida: Uber economy como plataforma virtual de prestación de servicios y su impacto en las relaciones laborales.» Revista Internacional y Comparada de Relaciones Laborales y Derecho del Empleo, 2017, Vol. V, n. ${ }^{\circ}$ 1, pp. 167-190.

34 Sentencia del Tribunal Central de Trabajo de Londres, de 28 de octubre de 2016 n. ${ }^{\circ}$ 2202551/2015, https://www.judiciary.gov.uk/judgments/mr-y-aslam-mr-j-farrar-andothers-v-uber/ (Última consulta el 12 de abril de 2017).

${ }_{35}$ TODOLÍ SIGNES, Adrián. «Comentario a la Sentencia que declara la laboralidad de los conductores de Uber en UK». Blog de Adrián Todolí. https://adriantodoli. com/2016/11/02/comentario-a-la-sentencia-que-declara-la-laboralidad-de-los-conductores-de-uber-en-uk/ (Última consulta el 4 de mayo de 2017). 
señala que Uber no hace publicidad en beneficio de ningún conductor en particular sino que Uber hace publicidad de su marca. Por tanto, difícilmente se puede hablar de coordinación entre empresas cuando se habla de Uber y los conductores.

En tercer lugar, el TCT considera difícilmente creíble que en Londres existan 30.000 pequeños empresarios unidos por una única plataforma. Así, admite que es fácilmente comprensible que si efectivamente esos 30.000 trabajadores fueran realmente autónomos, la empresa sería imposible de manejar.

En cuarto lugar, otro de los nuevos indicios de laboralidad se identifica con el control que hace la plataforma de la información clave para el desarrollo del negocio.

Por último, se destaca el hecho de que Uber se reserve el poder de modificar unilateralmente los términos del contrato.

Así, desde la publicación de esta sentencia, los conductores de Uber en Reino Unido dejarán de ser catalogados como autónomos para ser considerados trabajadores por cuenta ajena de la compañía, con las repercusiones que eso genera en términos de derechos laborales: derecho a vacaciones pagadas, al salario mínimo garantizado, jornadas laborales más reducidas...

b) Dictamen del Comisión de Trabajo de California ${ }^{36}$

El dictamen emitido por el Tribunal Superior de California en junio de 2015 resulta especialmente significativo por ser la primera resolución que abordó la cuestión de si existe o no relación jurídico laboral entre la empresa y sus conductores. Así, la Corte de California respondió a la denuncia presentada por un chófer de la compañía que exigía que Uber le pagase 4.152 dólares en atrasos y gastos durante las ocho semanas que condujo para la aplicación.

Esta sentencia fue la primera resolución en implementar el test de laboralidad, denominado en la jurisprudencia americana como «Borello test», para determinar la relación jurídica que vinculaba a las partes. Así, según la Comisión, el hecho de que Uber proporcione a sus conductores un teléfono móvil y esté pendiente de su actividad (para darlos de baja si no se conectan) puede considerarse ir más allá que el ejercer como una plataforma neutral que hace de intermediaria. Por tanto, el fallo determina la relación de laboralidad entre la demandante y la compañía. Así, si bien la sentencia no era vinculante y afectaba exclusivamente a las partes del litigio, en septiembre de 2015 se facultó a los conductores de la compañía para realizar demandas colectivas y

${ }^{36}$ Sentencia del Tribunal Superior de California, de 28 de octubre de 2016 n. ${ }^{\circ}$ CGC15-546378. https://es.scribd.com/doc/268911290/Uber-vs-Berwick (Última consulta el 12 de abril de 2017). 
así poder determinar su condición de empleados. Sin embargo, la compañía, temerosa de un vuelco a su modelo de negocio, logró sellar un acuerdo con más de 385000 conductores que le permite seguir considerándolos trabajadores independientes a cambio de más derechos y una indemnización global de 86 millones de euros. De esta forma, si bien el acuerdo mantiene la incertidumbre sobre el estatus de los trabajadores independientes, el objetivo principal no era otro sino disuadir las demandas en masa que puedan poner en peligro al fenómeno de la «gig-economy».

\subsection{Doctrinales}

Dentro de la doctrina laboralista española, cada vez son más numerosos los pronunciamientos a favor de la existencia de laboralidad entre Uber y sus conductores. Así, además del ya citado TODOLÍ SIGNES -quien sostiene que la figura del autónomo no encaja en la «gig-economy» puesto que desde las plataformas se controla y se dictan instrucciones a los trabajadores-, encontramos a otros autores como GINES I FABRELLAS y GÁLVEZ DURÁN ${ }^{37}$ quienes defienden que:

«la prestación de servicios en el marco de la organización empresarial Uber, la ausencia de una auténtica organización empresarial propia y autónoma y la ajenidad en los beneficios y riesgos de la actividad productiva confirman la existencia de una relación laboral, sin que la propiedad del vehículo o la libertad en la determinación del tiempo de trabajo y horario puedan apreciarse como relevantes para descartar la concurrencia de las notas de ajenidad y dependencia jurídica».

En esta misma línea, se posiciona CAVAS MARTINEZ ${ }^{38}$, afirmando que las notas tradicionales de laboralidad se presentan muy atenuadas en las prestaciones de servicios que tienen lugar en la «gig-economy», pero que ello no resulta obstáculo para que las plataformas puedan ser consideradas empleadoras de sus proveedores de servicio, si desde una conceptuación amplia y renovada de la nota de subordinación, adaptada a las características de estos nuevos modelos de negocio, se aprecian indicios significativos para entender que subsiste un poder de dirección o control de la plataforma sobre los prestadores de los servicios. Sobre este punto, también coincide SIERRA

${ }^{37}$ GINES I FABRELLAS, Anna; GALVEZ DURÁN, Sergi. «Sharing economy vs. Uber economy y las fronteras del Derecho del Trabajo: la (des)protección de los trabjadores en el nuevo entorno digital.» Revista para el análisis del Derecho, 2016, n. ${ }^{\circ}$ 1/2016, pp. 12-16.

38 CAVAS MÁRTINEZ, Faustino. «Las prestaciones de servicios a través de las plataformas informáticas de consumo colaborativo: un nuevo desafío para el Derecho del Trabajo.» Revista de Trabajo y Seguridad Social, 2017, n. ${ }^{\circ}$ 406, pp. 23-56. 
BÉNITEZ $^{39}$, quien además recalca la necesidad de adaptar los instrumentos de protección del trabajador a las nuevas realidades para poder realizar unas propuestas que garanticen los derechos fundamentales del trabajador en la era digital.

\section{Problemas de encaje del nuevo tipo de trabajadores en el concepto tradicional de contrato de trabajo}

Por tanto, de lo analizado hasta ahora, parece que existen argumentos suficientes para que los tribunales pudieran calificar a este nuevo tipo de prestadores personales de servicios a través de plataformas virtuales como trabajadores tradicionales cuyo empresario es la plataforma. En este sentido, la mayoría de la doctrina entiende que se está ante un nuevo tipo de subordinación derivado de las nuevas tecnologías donde la forma de controlar el trabajo es distinta, pero el resultado sigue siendo el mismo.

Sin embargo, a pesar de la posibilidad de encajar este tipo de prestación de servicios en el modelo tradicional de trabajo, se plantean una serie de problemas al respecto, puesto que el modelo de trabajo tradicional, no se asemeja en absoluto a muchos aspectos que hoy en día los modelos emergentes presentan. Con ello, tal y como señala RODRIGUEZ-PIÑERO ROYO «las consecuencias de una declaración de laboralidad pueden no resolver todas las necesidades normativas de éstos», por lo que se perfila necesaria una readaptación de los modelos tradicionales para respetar la esencia de estos modelos de negocio y de las nuevas relaciones de trabajo que traen consigo.

Así, conforme a los posibles problemas que la calificación como relación laboral «tradicional» podría conllevar, cabe destacar en primer lugar el hecho de que se aplicaría la normativa laboral en bloque, dando lugar a que, en ocasiones, se entienda la misma incompatible e incluso perjudicial para la condición del trabajador. Como se ha citado anteriormente, existen una serie de especialidades de este nuevo modelo de trabajo que difieren notablemente de las concepciones tradicionales del trabajo.

En segundo lugar, los trabajadores podrían ver afectada la autonomía jurídica que hasta entonces estos modelos de negocio les proporciona, entrometiéndose incluso en la forma de ejecutar su trabajo. Así, esto afectaría a una de las notas características de las plataformas como es el hecho de que sea el propio trabajador quien marca su ritmo de trabajo, su horario, su jornada, e incluso la forma de ejecutarlo.

39 SIERRA BÉNITEZ, Esperanza Macarena. «Los conductores privados de Uberpop: una nueva situación de anomia laboral». El Derecho del Trabajo y la Seguridad Social en la encrucijada: retos para la disciplina laboral, Laborum, Murcia, 2016 (en prensa). 
Finalmente, el hecho de entender que la prestación de servicios se ha llevado a cabo al margen de la ley (siempre y cuando se aprecie que los conductores de Uber no son autónomos, sino trabajadores con vinculación laboral) implicaría que hasta dicho momento, todas las actuaciones llevadas a cabo por Uber son consideradas ilegales, con las correspondientes sanciones y responsabilidades que ello conlleva ${ }^{40}$.

Por tanto, resulta necesario interesarse por las soluciones jurídicas que podrían establecerse para dar respuesta a los retos laborales que plantean estas plataformas de la «gig-economy».

\section{SOLUCIONES PARA DAR RESPUESTA A LOS NUEVOS RETOS LABORALES PLANTEADOS POR LA «GIG-ECONOMY»}

Dado que el encaje de los trabajadores de la «gig-economy» en la normativa laboral no sería cien por cien efectivo, cabe plantearse otras soluciones que permitan alcanzar la necesaria protección con la que deben contar los trabajadores de este sector. Para ello, se destacan dos soluciones antagónicas: por un lado, la autorregulación y, por otro lado, la intervención legislativa a través de la creación de una nueva relación laboral especial a tal efecto.

\section{La autorregulación}

Una de las soluciones que se presentan pasa por la autorregulación. Así, algunos autores defienden que la intervención legislativa debe esperar a que el fenómeno de la "gig-economy» termine por configurarse plenamente puesto que, de lo contrario, una regulación prematura podría impedir el desarrollo de una industria que se halla en su máximo apogeo. En este sentido, si bien la autorregulación podría plantearse como una opción exitosa en esta fase de crecimiento, en ella tendrían que tomar parte todos los intervinientes del sector, desde los «microtrabajadores» hasta los empresarios. En relación con los primeros, su participación se perfila complicada puesto que, como ya se ha recogido, las dinámicas de estas empresas hacen difícil la existencia de una representación unificada. Por tanto, excepto que se encuentre una manera eficaz de obtener la representación de los trabajadores rápidamente, cualquier forma de autorregulación vendrá a significar una regulación unilateral por parte de las plataformas virtuales, con las consecuencias que ello conllevaría en los derechos y garantías de los prestadores de servicios.

40 TODOLÍ SIGNES, Adrián. El trabajo de la era economía colaborativa. Primera edición. Valencia: Ed. Tirant lo Blanch, 2017, pp. 58-59. 


\section{Relación laboral especial}

De acuerdo con LOPEZ GANDÍA ${ }^{41}$, la idea que está latente en las relaciones laborales especiales es la flexibilidad, adecuación o modulación de la normativa laboral común en razón de las especialidades de los sectores que viene a laboralizar, contribuyendo así a perfilar las fronteras y a reducir las incertidumbres de las zonas grises del Derecho del Trabajo. Así, en el plano estatal, existen distintos casos en los que se ha propuesto una regulación diferente a la del trabajador común: personal de alta dirección, artistas en espectáculos públicos, deportistas profesionales, representantes de comercio...

En todos los supuestos, deben concurrir razones convincentes y suficientes para justificar desde el principio de igualdad el desigual tratamiento, de manera que la diferente regulación obedezca a situaciones verdaderamente desiguales.

Ciertamente, en el caso de las relaciones de los trabajadores de Uber, la relación laboral especial podría plantearse como solución puesto que, en este caso, la especialidad derivaría del marco organizativo en que se desarrolla la prestación y de la atenuación de las notas de ajenidad y dependencia, al manifestarse ambas de forma distinta al modelo tradicional. Precisamente, cabe destacar que la menor dependencia jurídica, como característica de relaciones laborales especiales es algo que ya ha servido de justificación en otras ocasiones, como por ejemplo en el caso de los representantes de comercio ${ }^{42}$.

De este modo, la relación laboral especial debería partir de su aplicación a aquellos trabajadores que prestaran servicios a través de plataformas virtuales específicas como las ya descritas. A partir de ahí, vistas las características del modelo de negocio, se debería tratar de proteger aquellos derechos básicos y aquellos otros que no impidieran el desarrollo normal de la industria, garantizando especialmente procedimientos de representación de los trabajadores con el objetivo de permitir, en definitiva, la autorregulación vía negociación colectiva.

Además, lo ideal sería que esta regulación permitiese ofrecer mecanismos para paliar la precariedad que, como hemos visto anteriormente, en ocasiones tienen que sufrir los trabajadores de la «gig-economy». Para ello, las cuestiones sobre las que la regulación debería pronunciarse necesariamente son las siguientes ${ }^{43}$ :

${ }^{41}$ LOPEZ GANDÍA, Juan. Las relaciones laborales especiales. Primera edición. Albacete: Ed. Bomarzo, 2008, p. 160.

${ }^{42}$ Ley 12/1992, de 27 de mayo, reguladora del contrato de agencia.

43 TODOLÍ SIGNES, Adrián. El trabajo de la era economía colaborativa. Primera edición. Valencia: Ed. Tirant lo Blanch, 2017, pp. 67-69. 
- Libertad de horarios y jornada: La regulación debería contemplar la libertad del trabajador para fijar su horario. El empresario, por su parte, debería poder fijar un número máximo de horas (pero no un mínimo) de trabajo semanal. Al mismo tiempo, la regulación debería establecer un máximo imperativo de jornada semanal con objeto de evitar sobrecarga de trabajo. Ese límite máximo de jornada debería ser global, es decir, se aplicaría por trabajador, computando todas las horas trabajadas en las diferentes plataformas virtuales en las que prestara servicios.

- Libertad para trabajar en varias plataformas: Con objeto de promocionar la libertad de entrada en el mercado, la regulación debería impedir que se pudieran firmar acuerdos de exclusividad con estos trabajadores.

- Responsabilidad por daños de los trabajadores: Como resultado de la falta de subordinación en la ejecución del trabajo, las empresas no podrían ser consideradas responsables de las acciones de los trabajadores. Por ello, alejándose del criterio clásico, los trabajadores deberían responder por las negligencias cometidas durante su prestación de servicios, incluyendo aquí accidentes de trabajo o daños a los clientes e incluso dañosa la marca o reputación de la plataforma virtual.

- Salario mínimo por el tiempo que prestan servicios: La empresa debería pagar un salario mínimo por el tiempo que efectivamente se esté prestando un servicio a un cliente. Sin embargo, en este caso la controversia se encuentra en qué sucede con los tiempos de espera. En el caso de Uber, los conductores deben estar activos y en movimiento para encontrar clientes, por lo que una solución equitativa para este tiempo puede ser considerarlo tiempo a disposición del empresario, pero improductivo. Este tiempo, según el artículo 30 del Estatuto de los Trabajadores, debe ser retribuido como tiempo de trabajo. No obstante, la normativa especial podría mantener vigente esta regulación, pero permitiendo que la negociación colectiva la modificara. De esta forma, los acuerdos colectivos podrían reducir la retribución de los tiempos de espera por debajo del mínimo o incluso eliminar toda retribución si lo consideran conveniente.

- Compensación de gastos: La normativa especial debería permitir que las empresas establecieran unos requisitos de bienes no consumibles que el trabajador debe poseer para poder formar parte de la plataforma. De esta forma, se podría exigir poseer teléfono, coche, ordenador para que estos bienes no sean pagados por la empresa. Sin embargo, los bienes consumibles que son necesarios para la ejecución de trabajo así como los gastos relacionados exclusivamente con la prestación del servicio deberían ser costeados por la compañía.

Por lo tanto, en caso de tramitarse esta regulación, esta normativa laboral especial no sólo permitiría proteger a los trabajadores de la «gig-economy» 
sino también adaptarse a los nuevos retos laborales que plantea el empleo de la era digital.

\section{CONCLUSIONES}

Primera. Tal y como plantean los órganos internacionales -tanto la UE, como la OIT-, existe un importante y creciente número de personas empleadas en las plataformas digitales de la «gig-economy». Así, la falta de intervención por parte del regulador en este campo está derivando en una creciente autorregulación, que, lejos de garantizar los derechos de los trabajadores que allí desarrollan sus servicios, está reforzando la capacidad de las plataformas para establecer de forma unilateral mecanismos de control que respondan a sus intereses particulares.

Segunda. Dichos mecanismos de control poco guardan en común con los sistemas tradicionales inspirados en el modelo taylorista. En este sentido, si bien el impacto de la digitalización en las relaciones laborales ha traído consigo la implantación de nuevos modelos de supervisión basados en un control automatizado (y, por ende, más exhaustivo) de los tiempos de trabajo de los trabajadores, lamentablemente no se vienen utilizando para garantizar los tiempos de descanso exigidos por la Ley.

Tercera. Analizadas las características de la relación laboral, tanto la jurisprudencia extranjera como la doctrina española coinciden en señalar la concurrencia de las notas de dependencia y ajenidad en la relación entre los conductores y la plataforma Uber. En este sentido, al basarse sobre estas dos notas la distinción entre trabajador por cuenta ajena y trabajador por cuenta propia, cabe concluir la existencia de laboralidad en dicha relación, estableciéndose una mayor protección para los conductores.

Cuarta. Sin embargo, las especialidades que presenta el trabajador de la «gig-economy» dificulta su encaje en la figura del trabajador por cuenta ajena. Así, la aplicación de la normativa laboral en bloque sería perjudicial para los intereses de los proveedores de servicios puesto que podría atentar contra aquellos parámetros que hacen precisamente de estos modelos de negocio su principal atractivo, como por ejemplo la mayor autonomía para la fijación de los horarios o la forma de ejecutar sus servicios.

Quinta. Dicho esto, entre las soluciones que plantea la doctrina española para proteger a los trabajadores de la «gig-economy» sin debilitar sus aspectos clave, se señalan dos alternativas: la autorregulación y la creación de una nueva relación laboral especial para los proveedores de servicios a través de plataformas digitales. La primera, como ya se ha constatado, se presenta como una opción poco alentadora puesto que la ausencia de representación colectiva de los trabajadores, inclinaría la autorregulación del lado de las plataformas, imponiendo éstas últimas las normas que mejor respondan a sus 
intereses económicos. Por otro lado, la opción de la regulación laboral especial se perfilaría como una alternativa idónea, siempre y cuando permita proteger los derechos de los trabajadores, planteando mejoras en negociación colectiva, y contribuyendo al desarrollo de la industria.

\section{BIBLIOGRAFIA}

Alarcón Caracuel, M. R. «La ajenidad en el mercado: un criterio definitorio del contrato de trabajo». Revista Española de Derecho del Trabajo, 1986, n. ${ }^{\circ} 28$, pp. 495-544.

AragüEz VALEnZuela, L. «Nuevos modelos de economía compartida: Uber economy como plataforma virtual de prestación de servicios y su impacto en las relaciones laborales.» Revista Internacional y Comparada de Relaciones Laborales y Derecho del Empleo, 2017, Vol. V, n. ${ }^{\circ}$ 1, pp. 167-190.

ARrieta Idiakez, F. J. Bases para el estudio del Derecho del trabajo. Tercera edición. Bilbao: Ed. Gomylex, 2014.

CAVAS MÁrtineZ, F. «Las prestaciones de servicios a través de las plataformas informáticas de consumo colaborativo: un nuevo desafío para el Derecho del Trabajo.» Revista de Trabajo y Seguridad Social, 2017, n. ${ }^{\circ}$ 406, pp. 23-56.

Cedrola Spremolla, G. «El trabajo en la era digital: Reflexiones sobre el impacto de la digitalización en el trabajo, la regulación laboral y las relaciones laborales.» Revista Internacional y Comparada de Relaciones Laborales y Derecho del Empleo, 2017, Vol. V, n. ${ }^{\circ}$ 1, pp. 8-38.

DAGNINO, E. «Uber law: perspectiva jurídico-laboral de la sharing/on-demand economy». Revista Internacional y Comparada de Relaciones Laborales y Derecho del Empleo. 2015, Vol. III, n. ${ }^{\circ}$ 3, pp. 1-32.

GINES I FABRELLAS, A. «Crowdsourcing: nueva modalidad de externalización productiva en el entorno digital. Reflexiones sobre su viabilidad jurídica.» Iuslabor, Asociación Catalana de Iuslaboralistas. Recuperado http://www.iuslabor.org/ jornades-i-seminaris/comunicacions/any-2017/ (Última consulta el 21 de abril de 2017).

Gines I FABRellas, A.; Galvez DuRÁn, S. «Sharing economy vs. Uber economy y las fronteras del Derecho del Trabajo: la (des)protección de los trabajadores en el nuevo entorno digital.» Revista para el análisis del Derecho, 2016, n. ${ }^{\circ} 1 / 2016$, pp. 12-16.

Hill, S. «The Future of Work in the Uber Economy. Creating a Safety Net in a Multi-Employer World». Boston Review: Political and Literary Forum, http:// bostonreview.net/us/steven-hill-uber-economy-individual-security-accounts (Última consulta 3 de mayo de 2017).

LACALLE, D. Trabajadores precarios, trabajadores sin derechos. Primera edición. Madrid: Ed. El Viejo Topo, 2009.

Lopez GandíA, J. Las relaciones laborales especiales. Primera edición. Albacete: Ed. Bomarzo, 2008.

Martin Valverde, A.; Rodríguez-Sañudo Gutiérrez, F.; García Murcia, J. Manual de Derecho del Trabajo. Vigesimocuarta edición. Madrid: Ed. Tecnos, 2015. 
OJEDA AviLÉs, A. «Ajenidad, dependencia o control: la causa del contrato.» Tribuna Social: Revista de Seguridad Social y Laboral, 2007, n. ${ }^{\circ}$ 195, pp. 13-24.

RODRIGUEZ-PIÑERO ROYO, M. «El trabajo 3.0 y la regulación laboral: por un enfoque creativo en su tratamiento legal». Departamento de Derecho del Trabajo de la Universidad de Sevilla. Universidad de Sevilla. http://grupo.us.es/iwpr/2016/09/07/ el-trabajo-3-0-y-la-regulacion-laboral-por-un-enfoque-creativo-en-su-tratamientolegal/ (Última consulta 12 de abril de 2017).

SIERRA BÉNITEZ, E. M. «Los conductores privados de Uberpop: una nueva situación de anomia laboral». El Derecho del Trabajo y la Seguridad Social en la encrucijada: retos para la disciplina laboral, Laborum, Murcia, 2016 (en prensa).

Todolí Signes, A. El trabajo de la era economía colaborativa. Primera edición. Valencia: Ed. Tirant lo Blanch, 2017.

«Comentario a la Sentencia que declara la laboralidad de los conductores de Uber en UK». Blog de Adrián Todolí. https://adriantodoli.com/2016/11/02/comentario-a-la-sentencia-que-declara-la-laboralidad-de-los-conductores-de-uberen-uk/ (Última consulta el 4 de mayo de 2017). 


\title{
IMPLICACIONES DE LA «GIG-ECONOMY» EN LAS RELACIONES LABORALES: EL CASO DE LA PLATAFORMA UBER
}

\section{Implications of the "gig-economy» in labor relations: The Case of the Uber Platform}

\author{
Maria Saenz de Buruaga Azcargorta \\ Universidad de Deusto \\ maria.saenzdeburuaga@gmail.com
}

http://dx.doi.org/10.18543/ed-67(1)-2019pp385-414

\section{Copyright}

Estudios de Deusto es una revista de acceso abierto, lo que significa que es de libre acceso en su in tegridad. Se permite su lectura, la búsqueda, descarga, distribución y reutilización legal en cualquier tipo de soporte sólo para fines no comerciales, sin la previa autorización del editor o el autor, siempre que la obra original sea debidamente citada y cualquier cambio en el original esté claramente indicado

Estudios de Deusto is an Open Access journal which means that it is free for full access, reading, search, download, distribution, and lawful reuse in any medium only for non-commercial purposes, without prior permission from the Publisher or the author; provided the original work is properly cited and any changes to the original are clearly indicated. 